\title{
Predictors of remission in the treatment of major depressive disorder: real-world evidence from a 6-month prospective observational study
}

This article was published in the following Dove Press journal:

Neuropsychiatric Disease and Treatment

22 January 2015

Number of times this article has been viewed

\author{
Diego Novick' \\ Jihyung Hong' \\ William Montgomery ${ }^{2}$ \\ Héctor Dueñas ${ }^{3}$ \\ Magdy Gado ${ }^{4}$ \\ Josep Maria Haro 5 \\ 'Eli Lilly and Company, Windlesham, \\ UK; ${ }^{2}$ Eli Lilly Australia Pty Ltd, West \\ Ryde, Australia; ${ }^{3}$ Eli Lilly de Mexico, \\ Mexico City, Mexico; ${ }^{4}$ Eli Lilly and \\ Company, Riyadh, Saudi Arabia; ${ }^{5}$ Parc \\ Sanitari Sant Joan de Déu, Fundació \\ Sant Joan de Déu, Universitat de \\ Barcelona, Barcelona, Spain
}

Background: This study examined potential predictors of remission among patients treated for major depressive disorder (MDD) in a naturalistic clinical setting, mostly in the Middle East, East Asia, and Mexico.

Methods: Data for this post hoc analysis were taken from a 6-month prospective, noninterventional, observational study that involved 1,549 MDD patients without sexual dysfunction at baseline in 12 countries worldwide. Depression severity was measured using the Clinical Global Impression of Severity and the 16-item Quick Inventory of Depressive Symptomatology Self-Report (QIDS-SR 16 ). Depression-related pain was measured using the pain-related items of the Somatic Symptom Inventory. Remission was defined as a QIDS-SR $_{16}$ score $\leq 5$. Generalized estimating equation regression models were used to examine baseline factors associated with remission during follow-up.

Results: Being from East Asia (odds ratio [OR] 0.48 versus Mexico; $P<0.001$ ), a higher level of depression severity at baseline (OR 0.77, $P=0.003$, for Clinical Global Impression of Severity; OR 0.92, $P<0.001$, for QIDS-SR ${ }_{16}$ ), more previous MDD episodes (OR 0.92, $P=0.007$ ), previous treatments/therapies for depression (OR $0.78, P=0.030$ ), and having any significant psychiatric and medical comorbidity at baseline (OR $0.60, P<0.001)$ were negatively associated with remission, whereas being male (OR 1.29, $=0.026)$ and treatment with duloxetine (OR 2.38 versus selective serotonin reuptake inhibitors, $P<0.001$ ) were positively associated with remission. However, the association between Somatic Symptom Inventory pain scores and remission no longer appeared to be significant in this multiple regression $(P=0.580),(P=0.008$ in descriptive statistics), although it remained significant in a subgroup of patients treated with selective serotonin reuptake inhibitors (OR 0.97, $P=0.023$ ), but not in those treated with duloxetine $(P=0.182)$.

Conclusion: These findings are largely consistent with previous reports from the USA and Europe. They also highlight the potential mediating role of treatment with duloxetine on the negative relationship between depression-related pain and outcomes of depression.

Keywords: antidepressant, duloxetine, selective serotonin reuptake inhibitors, Asia, Middle East, Mexico

\section{Introduction}

Major depressive disorder (MDD) is a highly prevalent condition that is associated with significant levels of disability, morbidity, and mortality. Most recently, the World Mental Health survey by the World Health Organization reported the lifetime prevalence of major depressive episode in 18 countries to range from $6.5 \%$ (People's Republic of China) and $6.6 \%$ (Japan) to $21.0 \%$ (France) and $19.2 \%$ (the USA). ${ }^{1}$ While the prevalence estimates were found to be greater in Western countries, emerging
Correspondence: Diego Novick Global Patient Outcomes and Real World Evidence (GPORWE), Eli Lilly and Company, Lilly Research Centre, Erl Wood Manor, Sunninghill Road, Windlesham, Surrey, GU20 6PH, UK Tel +4401276483832

$\mathrm{Fax}+4401276483192$

Email novick_diego@lilly.com 
evidence suggests that the prevalence rates in other parts of the world may have been underestimated. According to statistics from the Organisation for Economic Co-operation and Development (OECD) for instance, the suicide rates in East Asian countries like South Korea or Japan have been far above the OECD average. ${ }^{2}$ In addition, the 2010 Global Burden of Disease study revealed that the relative size of MDD burden, measured in disability-adjusted life years, was greater in the Middle East and North Africa than anywhere else, particularly for women in these regions. ${ }^{3,4}$ These findings reinforce the importance of treating MDD and related health problems as a public health priority, not only in the Western world, but in non-Western countries as well.

While treatment objectives have traditionally been centered around a reduction of symptoms, emerging evidence has shown that patients who experience clinically meaningful improvement but still have residual symptoms frequently experience significant levels of psychosocial impairment, and are also more vulnerable to relapse and recurrences of the disorder than patients who achieve full remission. ${ }^{5-7}$ Thus, there has been a growing emphasis on the importance of achieving remission in the treatment of MDD. This requires the development and implementation of treatment strategies that can increase the chances of achieving remission. Identifying predictors of remission in MDD is likely the first step, and can help guide clinical decision-making in heterogeneous patient populations.

Numerous studies have identified predictors of poor treatment outcomes of depression. However, most of these studies have employed "response" or "symptom improvement/worsening" rather than "remission" as an outcome, and have also been conducted in experimental settings with highly selected patient populations, possibly limiting generalizability to actual clinical practice. Two notable exceptions are the US Sequence Treatment Alternative to Relieve Depression (STAR*D) study $^{8}$ and the South Korean Clinical Research Centre for Depression study, ${ }^{9}$ both of which were carried out in "real world" settings. The former found that being Caucasian, being female, and having higher levels of education or income were positively associated with remission, whereas longer index episodes, more concurrent psychiatric and medical disorders, and lower functioning and quality of life at baseline were negatively associated with remission. This study also revealed the negative impact of painful physical symptoms, ${ }^{10}$ somatic symptomatology, ${ }^{11}$ concurrent alcohol and drug use, ${ }^{12}$ anxiety disorder ${ }^{13}$ and atypical features ${ }^{14}$ on remission. Similarly, the Clinical Research Centre for Depression study found the following potential predictors of remission: being female, no history of suicide attempt, and lower baseline severity of anxiety. A recent systematic review, which included a wide array of published studies on predictors of poor treatment outcomes of depression, ${ }^{15}$ reported similar findings, although it pointed out that the relationship between some of the factors (sex and severity of depression in particular) and treatment outcome could vary by class of antidepressant, treatment modality, and study outcomes. Nevertheless, given that most of the evidence comes from the USA and Europe, further research should examine whether these findings are also valid in other ethnic groups and cultures.

This study examined the baseline features associated with remission in the treatment of MDD for up to 6 months in a naturalistic clinical setting, mostly in the Middle East, East Asia, and Mexico, using data from a 6-month prospective observational study.

\section{Materials and methods Study design}

Data for this post hoc analysis were taken from a 6-month, international, prospective, noninterventional, observational study, primarily designed to examine treatment-emergent sexual dysfunction and other treatment outcomes among patients with MDD who were treated with either a selective serotonin reuptake inhibitor (SSRI) or a serotonin-norepinephrine reuptake inhibitor (SNRI) in actual clinical practice. A total of 1,647 patients were enrolled at 88 sites between November 15, 2007 and November 28, 2008. Of these, the 1,549 patients were classified as "sexually active patients without sexual dysfunction at study entry", and included in the study. The patients were drawn from the following countries and regions across the globe: East Asia (People's Republic of China [ $\mathrm{n}=205 ; 13.2 \%$ ], Hong Kong [ $=18 ; 1.2 \%$ ], Malaysia $[\mathrm{n}=33 ; 2.1 \%]$, the Philippines $[\mathrm{n}=113 ; 7.3 \%]$, Taiwan $[\mathrm{n}=199$; $12.8 \%$ ], Thailand $[n=17 ; 1.1 \%]$, and Singapore $[n=2 ; 0.1 \%])$, the Middle East (Saudi Arabia $[\mathrm{n}=179 ; 11.6 \%]$ and the United Arab Emirates $[n=135 ; 8.7 \%])$, Mexico $(n=591 ; 38.2 \%)$, and other regions (Israel $[n=9 ; 0.6 \%]$ and Austria $[n=48 ; 3.1 \%]$ ). This study followed the ethical standards of responsible local committees and the regulations of the participating countries, and was conducted in accordance with the ethical principles that have their origin in the Declaration of Helsinki and are consistent with Good Clinical Practice where applicable to a study of this nature. Ethical review board approval was obtained as necessary for observational studies wherever required by local law. All patients provided informed consent for the provision and collection of the data. Further details of the study design have been published elsewhere. ${ }^{16,17}$ 


\section{Study population}

Patients (on an outpatient basis) were eligible to participate in the study if they met the following inclusion criteria: presenting with an episode of MDD within the normal course of care, with MDD diagnosed according to the International Statistical Classification of Diseases Tenth Revision ${ }^{18}$ or Diagnostic and Statistical Manual of Mental Disorders Fourth Edition Text Revision (DSM-IV-TR) ${ }^{19}$ criteria; at least moderately depressed, defined by the Clinical Global Impression of Severity (CGI-S, with a score of $\geq 4$ ) ${ }^{20}$ initiating or switching to any available SSRI or SNRI antidepressant in any of the participating countries, in accordance with a treating psychiatrist's discretion; at least 18 years of age; sexually active (with partner or autoerotic activity, including during the 2 weeks prior to study entry) without sexual dysfunction, as defined by the Arizona Sexual Experience Scale (ASEX, ${ }^{21}$ ie, the criteria for sexual dysfunction: ASEX total score $\geq 19$, ASEX score $\geq 5$ on any item, or ASEX score $\geq 4$ on any three items); and not participating in another currently ongoing study. The study excluded patients who had: a history of treatment-resistant depression (defined as failure to respond to treatment with two antidepressants from different classes at therapeutic doses for $\geq 4$ weeks); a past or current diagnosis of schizophrenia, schizophreniform or schizoaffective disorder, bipolar disorder, dysthymia, mental retardation or dementia; or received any antidepressant within one week (one month for fluoxetine) prior to study entry, with the exception of patients receiving an ineffective treatment for whom immediate switch to an SSRI or SNRI antidepressant was considered to be the best treatment option.

Treatment decisions were made solely at the discretion of the treating psychiatrist, and were independent of study participation. Patients were not required to continue taking the medication initiated at baseline. Changes in medication and dosing, as well as use of concomitant medications and nonpharmacological therapies for the treatment of depression, were possible at any time as determined by the treating psychiatrist.

\section{Data collection and outcome assessment}

Data collection for the study occurred during visits within the normal course of care. The routine outpatient visit at which patients were enrolled served as the time for baseline data collection. Subsequent data collection was targeted at weeks 8,16 , and 24 following the baseline visit. Patient demographics and clinical history were recorded at the baseline assessment. Clinical severity of depression was assessed by treating psychiatrists at each visit using the CGI-S and 16-item Quick Inventory of Depressive Symptomatology Self-Report (QIDS-SR 16 ). ${ }^{22}$ Remission was defined as a QIDS-SR ${ }_{16}$ score $\leq 5$ at any time during the study period. This is more or less equivalent to a score of $\leq 7$ on the 17 -item Hamilton Depression Rating Scale. ${ }^{23,24}$ Depression-related pain severity was also measured using the pain-related items of the Somatic Symptom Inventory (SSI). ${ }^{25}$

\section{Statistical analysis}

This post hoc study examined baseline predictors of (timevarying) remission (ie, measured at each post-baseline visit) in the treatment of MDD. In order to minimize the confounding effect of medication over the course of treatment, we included only those patients who started with duloxetine or an SSRI as "monotherapy" $(\mathrm{n}=1,480)$, and analyzed patient observations up to the point where their initial medications were maintained $(\mathrm{n}=1,062$ [71.8\%] evaluable observations available at 24 weeks). Of the 1,480 patients, 1,194 were included in the final analysis because they were not in remission at baseline (ie, a QIDS-SR ${ }_{16}$ score $>5$ ) and also provided at least one post-baseline QIDS-SR ${ }_{16}$ assessment ( $\mathrm{n}=1,018$ [85.3\%] evaluable observations available at 24 weeks).

Baseline characteristics of patients who did $(n=939)$ or did not $(n=255)$ achieve remission during follow-up were first described and compared using the $t$-test (for continuous variables) and chi-squared test (for categorical variables).

Generalized estimating equation regression models with an exchangeable correlation structure were used to examine baseline factors associated with remission during follow-up, adjusting for visit numbers. The models included remission as a time-varying dependent variable, and also the following explanatory variables measured at baseline: age (years), sex (male/female), region (East Asia/the Middle East/Mexico/

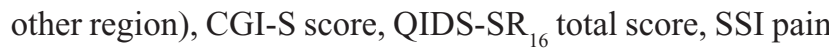
total score, and treatment (duloxetine/SSRI) at baseline. In addition, the following baseline variables were included if they appeared to be significant at $P<0.1$ in simple generalized estimating equation analyses (ie, one explanatory variable at once): age at first episode of MDD (years), body mass index $\left(\mathrm{kg} / \mathrm{m}^{2}\right)$, independent living (yes/no), living with a spouse/ partner (yes/no), educational attainment (up to primary school/secondary school or occupational program/up to university), employment status (full-time/economically inactive/ unemployed or part-time), number of MDD episodes in the 24 months prior to baseline, the receipt of any treatments/ therapies for depression in the 24 months prior to baseline (yes/no), and having any significant psychiatric and medical comorbidities (yes/no). 
These analyses were repeated for each treatment cohort (duloxetine and SSRIs) as well as for each region (Middle East, East Asia, and Mexico), except for "other region" due to a small sample size. All statistical analyses were carried out in SAS version 9.3 (SAS Institute, Cary, NC, USA).

\section{Results}

Of the 1,194 patients included in the final analysis, 42.4\% $(n=506)$ initiated duloxetine and the rest initiated an SSRI antidepressant $(57.6 \% ; \mathrm{n}=688)$ at baseline. The most common SSRIs prescribed at baseline were paroxetine (25.3\%), escitalopram (24.1\%), sertraline (20.5\%), and fluoxetine (19.3\%). The median daily doses of these medications at baseline were $60 \mathrm{mg}$ /day for duloxetine, $20.0 \mathrm{mg} /$ day for paroxetine, $10.0 \mathrm{mg}$ /day for escitalopram, $50.0 \mathrm{mg} /$ day for sertraline, and $20.0 \mathrm{mg}$ /day for fluoxetine.

A total of 939 patients (78.6\%) achieved remission during the 6-month follow-up period. Remission rates were higher in the Middle East (82.9\%) and Mexico (85.1\%) than in East Asia (69.7\%) and other regions, ie, Israel and Austria $(58.1 \%, P<0.001)$. It was also higher in patients who initiated duloxetine when compared with patients who initiated SSRIs $(84.0 \%$ versus $74.7 \%, P<0.001)$; a similar pattern was observed in each region (76.2\% versus $63.1 \%$ for East Asia, $91.0 \%$ versus $72.9 \%$ for the Middle East, and $90.8 \%$ versus $82.7 \%$ for Mexico, $P<0.05$ for all).

Table 1 describes the baseline demographics and clinical characteristics of patients by remission status during follow-up. Compared with those patients who did not achieve remission during follow-up, patients who achieved remission were more likely to be male, living in the Middle East or Mexico, living with a spouse/partner, have a higher level of education, and have initiated duloxetine (versus SSRI), but were less likely to have received treatments/therapies for depression in the past 24 months or to have significant concurrent psychiatric and medical conditions at baseline. In addition, these patients, on average, had slightly lower levels of clinical severity (measured by QIDS-SR ${ }_{16}$ ) and depression-related pain (measured by SSI pain) as well as a lower number of MDD episodes at baseline.

The results of multiple (generalized estimating equation) regression, which examined baseline patient characteristics associated with a time-varying remission over a 6-month period, are shown in Table 2. The associations listed above were largely maintained in this multiple regression analysis, except for SSI pain score. Patients who were male (odds ratio [OR] 1.29; $P=0.026$ ) and initiated duloxetine at baseline (OR $2.38 ; P<0.001)$ were more likely to achieve remission during follow-up. Meanwhile, those patients who were living in East Asia (OR 0.48 versus Mexico; $P<0.001$ ) or other regions (OR 0.38 versus Mexico; $P=0.004$ ), had higher CGI-S (OR $0.77 ; P=0.003$ ) and $\mathrm{QIDS}_{-\mathrm{SR}_{16}}$ (OR $\left.0.92 ; P<0.001\right)$ scores, had more MDD episodes during the past 24 months (OR $0.92 ; P=0.007)$, had received treatments/therapies for MDD in the past 24 months (OR $0.78 ; P=0.030$ ), and had significant psychiatric and medical comorbidities (OR 0.60; $P<0.001$ ) at baseline were less likely to achieve remission during follow-up. However, as already mentioned, the association between SSI pain scores and remission no longer appeared to be significant (OR 0.99; $P=0.580$ ), although it remained significant in a subgroup of patients who initiated on SSRIs (OR 0.97; $P=0.023$ ), but not in those initiated on duloxetine (OR 1.03; $P=0.182$; data not shown).

These analyses were repeated for each region (East Asia, the Middle East, and Mexico) as shown in Table 3. The results remained largely consistent in each of the subgroup analyses, but with fewer significant factors. As in the main analysis, the level of clinical severity (measured by CGI-S or QIDS-SR ${ }_{16}$ ) and type of medication initiated were consistently associated with remission during follow-up. Patients who were more severely ill were less likely to achieve remission (OR 0.68, $P=0.021$ [CGI-S] in East Asia; OR 0.88, $P=0.002$ [QIDS$\mathrm{SR}_{16}$ ] in the Middle East; OR 0.90, $P<0.001$ [QIDS-SR ${ }_{16}$ ] in Mexico), whereas those who initiated duloxetine (versus SSRIs) were more likely to achieve remission across the regions (OR 2.58, $P<0.001$ in East Asia; OR 2.78, $P<0.001$ in the Middle East; OR 1.95, $P<0.001$ in Mexico). Other factors significantly associated with (lower odds of) remission were the receipt of previous treatments/therapies for MDD (OR 0.69; $P=0.049$ ) in East Asia, more MDD episodes (OR $0.77 ; P<0.001)$ in the Middle East, and having significant psychiatric and medical comorbidities (OR 0.63; $P=0.004$ ) in Mexico. With regard to the association between SSI pain scores and remission, a pattern similar to that in the main analysis was observed in each of these subgroup analyses. However, unlike in the main analysis, the odds of achieving remission in patients with higher SSI pain scores were only numerically (ie, not significantly) lower than those with lower pain scores in SSRI-initiated patients.

\section{Discussion}

The present analysis, using data from a prospective observational study, identified baseline features associated with remission in MDD patients treated with duloxetine or an SSRI for up to 6 months in actual clinical practice settings, mostly in the Middle East, East Asia, and Mexico. Our 
Table I Patient characteristics by remission status during follow-up

\begin{tabular}{|c|c|c|c|}
\hline Baseline characteristic & No remission $(n=255)$ & Remission ( $n=939)$ & $P$-value \\
\hline Age (years), mean (SD) & $38.6(10.9)$ & $37.6(10.2)$ & 0.162 \\
\hline Sex & & & 0.029 \\
\hline Male, \% & 36.5 & 44.1 & \\
\hline Female, \% & 63.5 & 55.9 & \\
\hline Region, \% & & & $<0.001$ \\
\hline East Asia & 49.0 & 30.7 & \\
\hline Mexico & 29.8 & 46.2 & \\
\hline The Middle East & 16.1 & 21.2 & \\
\hline Other region & 5.1 & 1.9 & \\
\hline Ethnicity, \% & & & $<0.001$ \\
\hline East Asian & 49.4 & 30.8 & \\
\hline Hispanic & 29.8 & 45.8 & \\
\hline Mediterranean/Middle Eastern/North Africa descent Caucasian & 14.1 & 17.6 & \\
\hline European Caucasian & 4.3 & 2.7 & \\
\hline West Asian (Indian subcontinent) & 2.0 & 3.0 & \\
\hline African & 0.4 & 0.2 & \\
\hline Age at first symptoms of MDD (years), mean (SD) & $33.6(11.8)$ & $33.8(10.9)$ & 0.800 \\
\hline BMI $\left(\mathrm{kg} / \mathrm{m}^{2}\right)$, mean $(\mathrm{SD})$ & $24.4(4.7)$ & $24.8(4.3)$ & 0.302 \\
\hline Independent living, \% & 19.2 & 15.4 & 0.147 \\
\hline Living with a spouse/partner, $\%$ & 62.8 & 74.5 & $<0.001$ \\
\hline Educational attainment, \% & & & 0.009 \\
\hline Primary school or lower & 11.4 & 6.8 & \\
\hline Secondary school/occupational program & 47.1 & 42.7 & \\
\hline University or higher & 41.6 & 50.5 & \\
\hline Employment status, \% & & & 0.129 \\
\hline Full-time & 51.0 & 57.6 & \\
\hline Economically inactive & 28.2 & 25.9 & \\
\hline Unemployed/part-time & 20.8 & 16.5 & \\
\hline CGI-S, mean (SD) & $4.6(0.7)$ & $4.5(0.7)$ & 0.646 \\
\hline QIDS-SR $_{16}$, mean (SD) & $15.8(4.6)$ & I $4.2(4.3)$ & $<0.001$ \\
\hline SSI pain, mean (SD) & $15.4(5.5)$ & $14.4(4.8)$ & 0.008 \\
\hline Number of MDD in the past 24 months, mean (SD) & $1.7(2.6)$ & $1.2(1.7)$ & 0.005 \\
\hline Any treatments/therapies for depression in the past 24 months, \% & 52.6 & 39.1 & $<0.001$ \\
\hline Having significant comorbidities, \% & 28.9 & 22.4 & 0.031 \\
\hline Treatment with duloxetine (versus SSRI), \% & 31.8 & 45.3 & $<0.001$ \\
\hline
\end{tabular}

Notes: Data are presented as the percentage or the mean (standard deviation) as appropriate. Further details of the baseline characteristics of the study sample have been published elsewhere. ${ }^{16,17}$

Abbreviations: SD, standard deviation; BMI, body mass index; MDD, major depressive disorder; CGI-S, Global Impressions of Severity; QIDS-SR ${ }_{16}$, I6-item Quick Inventory of Depressive Symptomatology Self-Report; SSI pain, Somatic Symptom Inventory; SSRI, selective serotonin reuptake inhibitor.

results revealed relatively high rates of remission, ie, about $79 \%$ of patients achieved remission during follow-up, with the rate being higher in the Middle East (83\%) and Mexico $(85 \%)$ than in East Asia (70\%) and other regions (58\%). Several baseline features were found to be associated with higher remission rates, including being from the Middle East or Mexico, being male, having lower baseline severity of depression, fewer previous MDD episodes, no significant psychiatric and medical comorbidities, and being treated with duloxetine. Similar baseline features were identified as potential predictors of remission in subgroup analyses by region. These findings were generally consistent with those reported by the US STAR*D study, ${ }^{8}$ a large-scale prospective clinical trial carried out in a naturalistic clinical setting, confirming that this set of predictors of remission in MDD are mostly valid across different ethnic groups and cultures.

However, it should be noted that our remission rates, particularly in the Middle East and Mexico, were somewhat higher than those found in other studies. For instance, the STAR *D study reported a remission rate of $33 \%$ (defined as an exit score of $\leq 5$ on QIDS-SR ${ }_{16}$ ) among patients treated with citalopram (an SSRI) for up to 14 weeks, ${ }^{8}$ although it also noted a theoretical cumulative remission rate of $67 \%$ after four acute treatment steps (up to 14 weeks for each step). In addition, recent meta-analyses reported remission rates of $40 \%$ for duloxetine and $38 \%$ for SSRIs with pooled data from 
Table 2 Baseline factors associated with remission during follow-up (whole sample)

\begin{tabular}{|c|c|c|c|}
\hline Variables & Adjusted OR* & OR $95 \% \mathrm{Cl}$ & $\overline{P \text {-value }}$ \\
\hline Being older & 0.99 & $0.98-1.00$ & 0.184 \\
\hline Being male (versus female) & 1.29 & $1.03-1.62$ & 0.026 \\
\hline \multicolumn{4}{|l|}{ Region (versus Mexico) } \\
\hline East Asia & 0.48 & $0.37-0.63$ & $<0.00 \mathrm{I}$ \\
\hline Middle East & 0.82 & $0.60-1.12$ & 0.216 \\
\hline Other region & 0.38 & $0.20-0.73$ & 0.004 \\
\hline Living with spouse/partner & 1.23 & $0.96-1.58$ & 0.103 \\
\hline \multicolumn{4}{|l|}{ Educational attainment (versus primary school or lower) } \\
\hline Secondary school/occupational program & 1.03 & $0.70-|.5|$ & 0.885 \\
\hline University or higher & 1.28 & $0.86-1.90$ & 0.223 \\
\hline \multicolumn{4}{|l|}{ Employment status (versus full-time employment) } \\
\hline Economically inactive & 1.03 & $0.79-1.34$ & 0.823 \\
\hline Unemployed/part-time & 0.86 & $0.65-1.13$ & 0.278 \\
\hline Higher CGI-S score & 0.77 & $0.65-0.91$ & 0.003 \\
\hline Higher QIDS-SR ${ }_{16}$ score & 0.92 & $0.90-0.95$ & $<0.00$ I \\
\hline Higher SSI pain score & 0.99 & $0.97-1.02$ & 0.580 \\
\hline More MDD episodes in the past 24 months & 0.92 & $0.86-0.98$ & 0.007 \\
\hline Any treatment/therapies for MDD in the past 24 months & 0.78 & $0.63-0.98$ & 0.030 \\
\hline Having significant comorbidities & 0.60 & $0.47-0.76$ & $<0.00 \mathrm{I}$ \\
\hline Treatment with duloxetine (versus SSRI) & 2.38 & $1.90-2.98$ & $<0.001$ \\
\hline
\end{tabular}

Notes: *This multiple regression included remission as a time-varying variable during follow-up, and was also adjusted for visit numbers.

Abbreviations: $\mathrm{Cl}$, confidence interval; OR, odds ratio; MDD, major depressive disorder; CGI-S, Global Impressions of Severity; QIDS-SR ${ }_{16}$, I6-item Quick Inventory of Depressive Symptomatology Self-Report; SSI pain, Somatic Symptom Inventory; SSRI, selective serotonin reuptake inhibitor.

six 8-week clinical trials (defined as an exit score of $\leq 5$ on the 17-item Hamilton Depression Rating Scale), ${ }^{26} 52 \%$ for mirtazapine (a newer antidepressant with noradrenergic and specific serotonergic effects) and $47 \%$ for SSRIs with clinical trials lasting at least 8 weeks. ${ }^{27}$ Although it is not clear why our remission rates were higher than those rates published, one possible reason can be found in the characteristics of our patient sample, ie, this was an observational study designed primarily to assess the frequency of treatmentemergent sexual dysfunction in the treatment of MDD and thus included only those patients who were sexually active without sexual dysfunction at baseline. It is possible that such

Table 3 Baseline factors significantly associated with remission in each region*

\begin{tabular}{|c|c|c|c|}
\hline Variables & Adjusted OR & OR 95\% Cl & $P$-value \\
\hline \multicolumn{4}{|l|}{ East Asia } \\
\hline Being older & 1.00 & $0.98-1.02$ & 0.888 \\
\hline Being male (versus female) & 1.00 & $0.70-1.42$ & 0.983 \\
\hline Higher CGI-S score & 0.68 & $0.50-0.94$ & 0.021 \\
\hline Any treatment/therapies for MDD in the past 24 months & 0.69 & $0.47-0.998$ & 0.049 \\
\hline Treatment with duloxetine (versus SSRI) & 2.58 & I.77-3.76 & $<0.001$ \\
\hline \multicolumn{4}{|l|}{ Middle East } \\
\hline Being older & 1.00 & $0.97-1.03$ & 0.892 \\
\hline Being male (versus female) & 1.83 & $0.98-3.40$ & 0.058 \\
\hline Higher QIDS-SR ${ }_{16}$ score & 0.88 & $0.8 \mathrm{I}-0.95$ & 0.002 \\
\hline More MDD episodes in the past 24 months & 0.77 & $0.66-0.90$ & $<0.001$ \\
\hline Treatment with duloxetine (versus SSRI) & 2.78 & $1.66-4.66$ & $<0.00$ I \\
\hline \multicolumn{4}{|l|}{ Mexico } \\
\hline Being older & 1.00 & $0.98-1.01$ & 0.491 \\
\hline Being male (versus female) & 1.29 & $0.9 \mid-1.84$ & 0.157 \\
\hline Higher QIDS-SR 16 score & 0.90 & $0.86-0.93$ & $<0.00$ I \\
\hline Having significant comorbidities & 0.63 & $0.46-0.87$ & 0.004 \\
\hline Treatment with duloxetine (versus SSRI) & 1.95 & $1.37-2.78$ & $<0.001$ \\
\hline
\end{tabular}

Notes: *Only significant factors are presented in this table, except for age and sex. This multiple regression included remission as a time-varying variable during follow-up, and was also adjusted for visit numbers.

Abbreviations: $\mathrm{Cl}$, confidence interval; OR, odds ratio; MDD, major depressive disorder; CGI-S, Global Impressions of Severity; QIDS-SR ${ }_{16}$, I6-item Quick Inventory of Depressive Symptomatology Self-Report; SSRI, selective serotonin reuptake inhibitor. 
patients could have a better MDD prognosis than those with sexual dysfunction. Notably, rates of antidepressant switch or augmentation in this observational study were also found to be very low $(0.6 \%-2 \%) .{ }^{16}$ However, this still does not explain why patients in the Middle East and those in Mexico had higher remission rates. We may first need to understand whether patients who sought help and were treated for psychiatric illness could represent the general MDD populations in the regions, particularly in the Middle East, where mental illness and the use of mental health services carry an intense and enduring stigma. ${ }^{28,29}$

Nevertheless, our findings regarding factors associated with remission were generally consistent with those of the STAR*D study. ${ }^{8}$ The exceptions were the impacts of sex and depression-related pain, as well as the superiority of duloxetine over SSRIs (not examined in the STAR*D) in achieving remission. First of all, our study found a positive association between being male and remission, probably mainly influenced by the Middle East, which was in fact the only region showing a significant association between these two factors at the regional level, albeit marginal at the $10 \%$ significance level $(P=0.058)$. This observation contrasts with the findings of the STAR*D, which reported higher remission rates among females. The impact of sex on remission is not conclusive in the literature either. A recent systematic review on predictors of treatment outcomes in patients with depression reported mixed findings for the association between sex and treatment outcome. ${ }^{15}$ The review identified only one study showing a modest sex effect out of the seven studies involving tricyclic antidepressants. As in our study, a higher proportion of males were found to respond to imipramine, ${ }^{30}$ whereas females were found to be more likely than males to respond to SSRIs. ${ }^{15}$ Although the association between sex and remission has not been well established as yet, our findings are consistent with the recent report from the Global Burden of Disease 2010 study, which identified the highest burden of MDD to be in the Middle East and North African regions, particularly among females. Taken together, these findings imply that the stigma attached to mental illness as well as psychological distress is more severe in Arabic women, potentially resulting in poorer outcomes. More effort should be made to better understand the mental health needs of Arabic women as well as cultural and contextual factors associated with their mental health.

Although depression-related pain initially did not appear to be a potential predictor of remission in the whole sample, the findings of our subgroup analyses by treatment cohort are in line with those of previous studies. Clinical trials and case reports have shown that treatment with antidepressants may help decrease pain in patients with depression and in those with pain alone, and that the existing evidence suggests that SNRIs, including duloxetine, may be more effective than SSRIs for the relief of painful physical symptoms. ${ }^{10,31-37}$ Consistent with these results, patients taking duloxetine achieved the same level of remission independent of pain severity at baseline in our subgroup analyses, whereas this was not found for the subgroup of patients taking SSRIs. That is, patients taking SSRIs who had a higher level of pain severity at baseline did not achieve the same level of remission as those taking SSRIs who had a lower level of pain severity at baseline, confirming the negative impact of pain on achieving remission in this cohort (but not in the duloxetine cohort nor in the whole sample).

This may in part explain our finding that duloxetinetreated patients were more likely than SSRI-treated patients to achieve remission, especially given that $53 \%$ of our patients had painful physical symptoms at baseline (defined as an average score $\geq 2$ for pain-related items on the SSI). In addition, there is limited evidence that SNRIs might be more efficacious than SSRIs in those patients with more severe MDD ${ }^{26}$ and our study included at least moderately ill patients (defined as a CGI-S score $\geq 4$ ). Nevertheless, "no sexual dysfunction", another inclusion criterion, makes it hard to understand the impact of clinical severity on different treatment outcomes. Further research is clearly required to investigate the relationships and interactions between depression-related pain, type of treatment, severity of illness, presence/absence of sexual dysfunction, and outcomes of treatment for depression.

Our study has several limitations that should be taken into account when interpreting these results. First, as mentioned earlier, this study included only patients who were sexually active without sexual dysfunction at baseline because its primary objective was to assess the frequency of treatmentemergent sexual dysfunction in the treatment of MDD. Sexual dysfunction has been reported to be two to three times more prevalent in patients with depression compared with the general population, ${ }^{38,39}$ and thus our findings may not be immediately generalizable to MDD patients as a whole. Further research is warranted to examine whether these findings can be replicated in MDD patients without such inclusion criteria. Nevertheless, our findings are generally in line with those of the STAR*D study. Second, although our study prospectively assessed the level of depression severity (and thereby remission as well) in a "real-world" setting, the associations found in our study may not imply causal relationships given 
its observational design. Third, although this observational study included more than 1,000 patients, these patients may not be representative of those with MDD in each region. Finally, a multiplicity of analyses was not adjusted for in these exploratory analyses, given that this study was designed to be hypothesis-generating, not hypothesis-testing.

\section{Conclusion}

The results of this 6-month prospective observational study identify a number of potential predictors of remission in the treatment of MDD in the Middle East, East Asia, and Mexico, where such information is very limited. Similar to several previous studies in the USA and Europe, a higher level of depression severity, more previous MDD episodes, previous treatments/therapies for depression, and having significant psychiatric or medical comorbidity were negatively associated with remission, whereas being male and treatment with duloxetine (versus SSRIs) were positively associated with remission. We also found that patients taking duloxetine, unlike those taking SSRIs, achieved the same level of remission independently of their pain severity at baseline. This suggests that the negative impact of pain on remission may be mitigated somewhat by treatment with duloxetine, which may in turn help to explain the higher remission rates found for duloxetine-treated patients. Further research is needed to improve our understanding of the complex relationships between depression-related pain, treatment with antidepressants, and outcomes of depression. Overall, these findings may help to guide clinical decisionmaking in MDD for heterogeneous patient populations, particularly in non-Western countries.

\section{Disclosure}

This study was funded by Eli Lilly and Company. DN, WM, and HD are employees of Eli Lilly and Company. MG is a former employee of Eli Lilly and Company. JH is a consultant for Eli Lilly and Company. JMH has acted as a consultant, received grants, and acted as a speaker in activities sponsored by Astra-Zeneca, Eli Lilly and Company, Glaxo-SmithKline, and Lundbeck. The authors report no other conflicts of interest in this work.

\section{References}

1. Bromet E, Andrade LH, Hwang I, et al. Cross-national epidemiology of DSM-IV major depressive episode. BMC Med. 2011;9:90.

2. Organisation for Economic Cooperation and Development. ECD Health Statistics 2013. Available from: http://stats.oecd.org/index. aspx?DataSetCode=HEALTH_STAT. Accessed December 10, 2014.

3. Murray CJ, Vos T, Lozano R, et al. Disability-adjusted life years (DALYs) for 291 diseases and injuries in 21 regions, 1990-2010: a systematic analysis for the Global Burden of Disease Study 2010. Lancet. 2012;380(9859):2197-2223.
4. Institute for Health Metrics and Evaluation. Global Burden of Diseases, Injuries, and Risk Factors Study 2010 (GBD 2010). Seattle, WA, USA: Institute for Health Metrics and Evaluation; 2013.

5. Nierenberg AA, Husain MM, Trivedi MH, et al. Residual symptoms after remission of major depressive disorder with citalopram and risk of relapse: a STAR*D report. Psychol Med. 2010;40(1):41-50.

6. Judd LL, Paulus MJ, Schettler PJ, et al. Does incomplete recovery from first lifetime major depressive episode herald a chronic course of illness? Am J Psychiatry. 2000;157(9):1501-1504.

7. Paykel ES, Ramana R, Cooper Z, Hayhurst H, Kerr J, Barocka A. Residual symptoms after partial remission: an important outcome in depression. Psychol Med. 1995;25(6):1171-1180.

8. Trivedi MH, Rush AJ, Wisniewski SR, et al. Evaluation of outcomes with citalopram for depression using measurement-based care in STAR*D: implications for clinical practice. Am J Psychiatry. 2006;163(1):28-40.

9. Kim JM, Kim SW, Stewart R, et al. Predictors of 12-week remission in a nationwide cohort of people with depressive disorders: the CRESCEND study. Hum Psychopharmacol. 2011;26(1):41-50.

10. Leuchter AF, Husain MM, Cook IA, et al. Painful physical symptoms and treatment outcome in major depressive disorder: a STAR*D (Sequenced Treatment Alternatives to Relieve Depression) report. Psychol Med. 2010;40(2):239-251.

11. Silverstein B, Patel P. Poor response to antidepressant medication of patients with depression accompanied by somatic symptomatology in the STAR*D Study. Psychiatry Res. 2011;187(1-2):121-124.

12. Davis LL, Wisniewski SR, Howland RH, et al. Does comorbid substance use disorder impair recovery from major depression with SSRI treatment? An analysis of the STAR*D level one treatment outcomes. Drug Alcohol Depend. 2010;107(2-3):161-170.

13. Fava M, Rush AJ, Alpert JE, et al. Difference in treatment outcome in outpatients with anxious versus nonanxious depression: a STAR*D report. Am J Psychiatry. 2008;165(3):342-351.

14. Stewart JW, McGrath PJ, Fava M, et al. Do atypical features affect outcome in depressed outpatients treated with citalopram? Int $J$ Neuropsychopharmacol. 2010;13(1):15-30.

15. Van HL, Schoevers RA, Dekker J. Predicting the outcome of antidepressants and psychotherapy for depression: a qualitative, systematic review. Harv Rev Psychiatry. 2008;16(4):225-234.

16. Duenas H, Brnabic AJ, Lee A, et al. Treatment-emergent sexual dysfunction with SSRIs and duloxetine: effectiveness and functional outcomes over a 6-month observational period. Int J Psychiatry Clin Pract. 2011;15(4):242-254.

17. Duenas H, Lee A, Brnabic AJ, et al. Frequency of treatment-emergent sexual dysfunction and treatment effectiveness during SSRI or duloxetine therapy: 8-week data from a 6-month observational study. Int J Psychiatry Clin Pract. 2011;15(2):80-90.

18. World Health Organization. The ICD-10 Classification of Mental and Behavioural Disorders. Clinical Descriptions and Diagnostic Guidelines. Geneva, Switzerland: World Health Organization; 1992. Available from: http://www.who.int/classifications/icd/en/bluebook. pdf. Accessed December 10, 2014.

19. American Psychiatric Association. Diagnostic and Statistical Manual of Mental Disorders (Fourth Edition Text Revision). Washington, DC, USA: American Psychiatric Publishing; 2000.

20. Guy W. ECDEU Assessment Manual for Psychopharmacology (Revised). Bethesda, MD, USA: US Department of Health, Education and Welfare; 1976.

21. McGahuey CA, Gelenberg AJ, Laukes CA, et al. The Arizona Sexual Experience Scale (ASEX): reliability and validity. $J$ Sex Marital Ther. 2000;26(1):25-40.

22. Rush AJ, Trivedi MH, Ibrahim HM, et al. The 16-Item Quick Inventory of Depressive Symptomatology (QIDS), clinician rating (QIDS-C), and self-report (QIDS-SR): a psychometric evaluation in patients with chronic major depression. Biol Psychiatry. 2003;54(5):573-583.

23. Ciraulo DA, Evans JA, Qiu WQ, Shader RI, Salzman C. Pharmacotherapy of depression. In: Ciraulo DA, Shader RI, editors. Clinical Pharmacology and Therapeutics of Antidepressants. 2nd ed. New York, NY, USA: Humana Press; 2011. 
24. Hamilton M. A rating scale for depression. J Neurol Neurosurg Psychiatry. 1960;23:56-62.

25. Kroenke K, Spitzer RL, Williams JB, et al. Physical symptoms in primary care. Predictors of psychiatric disorders and functional impairment. Arch Fam Med. 1994;3(9):774-779.

26. Thase ME, Pritchett YL, Ossanna MJ, Swindle RW, Xu J, Detke MJ. Efficacy of duloxetine and selective serotonin reuptake inhibitors: comparisons as assessed by remission rates in patients with major depressive disorder. J Clin Psychopharmacol. 2007;27(6):672-676.

27. Thase ME, Nierenberg AA, Vrijland P, van Oers HJJ, Schutte A-J, Simmons JH. Remission with mirtazapine and selective serotonin reuptake inhibitors: a meta-analysis of individual patient data from 15 controlled trials of acute phase treatment of major depression. Int Clin Psychopharmacol. 2010;25(4):189-198.

28. Shrivastava A, Johnston M, Bureau Y. Stigma of Mental Illness-2: noncompliance and intervention. Mens Sana Monogr. 2012;10(1):85-97.

29. Hamdan A. Mental health needs of Arab women. Health Care Women Int. 2009;30(7):595-613.

30. Kornstein SG, Schatzberg AF, Thase ME, et al. Gender differences in treatment response to sertraline versus imipramine in chronic depression. Am J Psychiatry. 2000;157(9):1445-1452.

31. Krell HV, Leuchter AF, Cook IA, Abrams M. Evaluation of reboxetine, a noradrenergic antidepressant, for the treatment of fibromyalgia and chronic low back pain. Psychosomatics. 2005;46(5):379-384.
32. Sindrup SH, Otto M, Finnerup NB, Jensen TS. Antidepressants in the treatment of neuropathic pain. Basic Clin Pharmacol Toxicol. 2005; 96(6):399-409.

33. Atkinson JH, Slater MA, Capparelli EV, et al. Efficacy of noradrenergic and serotonergic antidepressants in chronic back pain: a preliminary concentration-controlled trial. J Clin Psychopharmacol. 2007; 27(2):135-142.

34. Jann MW, Slade JH. Antidepressant agents for the treatment of chronic pain and depression. Pharmacotherapy. 2007;27(11):1571-1587.

35. Fava M, Mallinckrodt CH, Detke MJ, Watkin JG, Wohlreich MM. The effect of duloxetine on painful physical symptoms in depressed patients: do improvements in these symptoms result in higher remission rates? $J$ Clin Psychiatry. 2004;65(4):521-530.

36. Greco T, Eckert G, Kroenke K. The outcome of physical symptoms with treatment of depression. J Gen Intern Med. 2004;19(8):813-818.

37. Perahia DGS, Quail D, Desaiah D, Montejo AL, Schatzberg AF. Switching to duloxetine in selective serotonin reuptake inhibitor non- and partial-responders: effects on painful physical symptoms of depression J Psychiatr Res. 2009;43(5):512-518.

38. Angst J. Sexual problems in healthy and depressed persons. Int Clin Psychopharmacol. 1998;13 Suppl 6:S1-S4.

39. Bonierbale M, Lancon C, Tignol J. The ELIXIR study: evaluation of sexual dysfunction in 4,557 depressed patients in France. Curr Med Res Opin. 2003;19(2):114-124.
Neuropsychiatric Disease and Treatment

\section{Publish your work in this journal}

Neuropsychiatric Disease and Treatment is an international, peerreviewed journal of clinical therapeutics and pharmacology focusing on concise rapid reporting of clinical or pre-clinical studies on a range of neuropsychiatric and neurological disorders. This journal is indexed on PubMed Central, the 'PsycINFO' database and CAS,

\section{Dovepress}

and is the official journal of The International Neuropsychiatric Association (INA). The manuscript management system is completely online and includes a very quick and fair peer-review system, which is all easy to use. Visit http://www.dovepress.com/testimonials.php to read real quotes from published authors. 